\title{
Resonant damping of kink oscillations of thin cooling and expanding coronal magnetic loops
}

\author{
A. A. Shukhobodskiy ${ }^{1,2}$, M. S. Ruderman ${ }^{1,3}$, and R. Erdélyi ${ }^{1,4}$ \\ ${ }^{1}$ Solar Physics and Space Plasma Research Centre ( $\left.\mathrm{SP}^{2} \mathrm{RC}\right)$, University of Sheffield, Hicks Building, Hounsfield Road, Sheffield S3 \\ 7R, UK \\ e-mail: m.s.ruderman@sheffield.ac.uk \\ 2 ITMO University, Kronverkskii ave 49, 197101 Saint-Petersburg, Russia \\ 3 Space Research Institute (IKI), Russian Academy of Sciences, Moscow 117810, Russia \\ ${ }^{4}$ Dept of Astronomy, Eötvös L. University, Pázmány P. sétány 1/A, Budapest 1117, Hungary
}

Received 25 June 2018 / Accepted 12 September 2018

\begin{abstract}
We have considered resonant damping of kink oscillations of cooling and expanding coronal magnetic loops. We derived an evolutionary equation describing the dependence of the oscillation amplitude on time. When there is no resonant damping, this equation reduces to the condition of conservation of a previously derived adiabatic invariant. We used the evolutionary equation describing the amplitude to study the competition between damping due to resonant absorption and amplification due to cooling. Our main aim is to investigate the effect of loop expansion on this process. We show that the loop expansion acts in favour of amplification. We found that, when there is no resonant damping, the larger the loop expansion the faster the amplitude growths. When the oscillation amplitude decays due to resonant damping, the loop expansion reduces the damping rate. For some values of parameters the loop expansion can fully counterbalance the amplitude decay and turn the amplitude evolution into amplification.
\end{abstract}

Key words. hydrodynamics - magnetohydrodynamics (MHD) - plasmas - waves - methods: analytical - Sun: corona

\section{Introduction}

After transverse coronal loop oscillations were first observed by TRACE in 1998 and reported by Aschwanden et al. (1999) and Nakariakov et al. (1999), they received ample attention in the solar physics community. Since then, these oscillations were routinely observed during various space missions (see e.g. Erdélyi \& Taroyan 2008; Duckenfield et al. 2018; Su et al. 2018; Abedini 2018, and references therein).

One important property of transverse coronal loop oscillations is that they are strongly damped with the damping time being comparable with the oscillation period. At present, a generally accepted mechanism of this damping is resonant absorption. It was suggested by Hollweg \& Yang (1988) ten years before the first observation of transverse coronal loop oscillations that, if these coronal loop oscillations exist, they can be strongly damped by resonant absorption. Hollweg \& Yang (1988) studied resonant absorption using planar geometry, but then translated their result to the cylindrical geometry and obtained the correct expression for the decrement in the thin tube approximation. Later Goossens et al. (1992) studied the damping of kink oscillations of magnetic flux tubes due to resonant absorption in the general case. Ruderman \& Roberts (2002) applied the theory of wave damping due to resonant absorption to the first observation of coronal loop kink oscillations. They showed that the observed damping of these oscillations can be used to obtain information about the internal structure of coronal magnetic loops. Ruderman \& Roberts (2002) modelled a coronal loop as a magnetic tube consisting of an internal core of radius $R$ and a transitional or boundary layer of thickness $\ell$ between the dense core plasma and the rarefied surrounding plasma. They obtained that the decrement is proportional to $\ell / R$. Using the data on the oscillation damping reported by Nakariakov et al. (1999) they obtained that $\ell / R=$ 0.23 . Goossens et al. (2002) used observations of eleven cases of damped kink oscillations of coronal magnetic loops to estimate $\ell / R$. They obtained values of $\ell / R$ between 0.16 and 0.49 . Since then, observations of damped coronal loop oscillations are continuously used to obtain information on the loop internal structure (e.g. Ruderman \& Erdélyi 2009; Goossens et al. 2011).

In the first studies of kink oscillations of coronal magnetic loops, a very simple model of a homogeneous magnetic cylinder was used (e.g. Ryutov \& Ryutova 1976; Edwin \& Roberts 1983). In this model, the tube has a sharp boundary, so it does not describe resonant absorption. To describe resonant absorption, this model was modified by including a transitional layer at the tube boundary. Later, more realistic models of coronal loops were studied. In particular the variation of the plasma density along the tube was taken into account. Dymova \& Ruderman (2006) investigated the resonant damping of kink oscillations of a magnetic tube with such density variation. The main result that they obtained is the following: if the ratio of densities in the tube core and in the surrounding plasma is constant, and the ratio of density inside the boundary layer and in the tube core does not vary along the tube, then the ratio of the damping time and oscillation period is not affected by the density variation along the tube.

Although the coronal loop expansion is relatively small, the ratio of the loop cross-section radii at the apex and at the foot-points still can be about 1.5 (Klimchuk 2000; Watko \& Klimchuk 2000). On the other hand, in the chromosphere the expansion of vertical magnetic flux tubes can be 
as large as a few hundred (e.g. Tsuneta et al. 2008). Hence, the account of the magnetic flux tube expansion is important. Ruderman et al. (2008) and Verth \& Erdélyi (2008) derived the equation describing kink oscillations of an expanding magnetic flux tube. They considered a magnetic flux tube with a sharp boundary meaning that the equation that they derived does not describe resonant damping. Ruderman et al. (2017) generalised this derivation to include a siphon flow, temporal variation of the plasma parameters, and a transitional layer at the tube boundary.

Observations show that very often oscillating coronal loops are in a highly dynamic state. In particular, they can cool quickly with a characteristic cooling time of the order of a few periods of kink oscillation (e.g. Aschwanden \& Terradas 2008). Morton \& Erdélyi $(2009,2010)$ found that cooling results in the decrease of the period of the coronal loop kink oscillations, while similar results were found by Al-Ghafri et al. (2014) for longitudinal oscillations. Ruderman (2011b) showed that cooling causes the amplification of coronal loop kink oscillations. Ruderman (2011a) studied the competition between cooling and resonant damping. He showed that this competition can result in the existence of kink oscillations with the amplitude not varying in time. Recently, Ruderman et al. (2017) studied the effect of tube expansion on kink oscillations of cooling coronal loops.

Ruderman et al. (2017) considered a magnetic tube with the sharp boundary thus eliminating the effect of resonant absorption. In this article we study the effect of resonant absorption on kink oscillations of cooling and expanding coronal loops. The paper is organised as follows. In the next section we formulate the problem and write down the governing equations. In Sect. 3 we consider kink oscillations of coronal loop with slowly changing density and derive the equation describing the evolution of the oscillation amplitude. In Sect. 4 we study kink oscillations of cooling coronal loops with the density exponentially decreasing with the height. Section 5 contains the summary of obtained results and our conclusions.

\section{Problem formulation}

We consider kink oscillations of a thin straight magnetic flux tube with circular cross-section. The assumption that the tube is thin, that is its radius is much smaller than the wavelength, is usually satisfied for kink waves in magnetic tubes in the solar atmosphere. For example, the typical wave length of oscillations in coronal magnetic loops is of the order of the loop length, which is by about two orders of magnitude larger than the crosssection radius. The cross-section radius varies along the tube. The tube consists of a core region where the plasma density only weakly varies in the radial direction, and the transitional region where the density quickly decreases from its value in the core region to that in the external plasma. A sketch of the unperturbed configuration is shown in Fig. 1.

Below, we use cylindrical coordinates $r, \phi, z$. In these coordinates the equilibrium density is given by

$\rho=\left\{\begin{array}{l}\rho_{\mathrm{i}}(t, r, z), \quad 0 \leq r \leq R(z)(1-l / 2), \\ \rho_{t}(t, r, z), \quad R(z)(1-l / 2) \leq r \leq R(z)(1+l / 2), \\ \rho_{\mathrm{e}}(t, r, z), \quad r \geq R(z)(1+l / 2),\end{array}\right.$

where $R(z)$ is the tube radius, and $l R(z)$ is the thickness of the transitional layer. There is also the plasma flow $\boldsymbol{U}=$ $\left(U_{r}(t, r, z), 0, U_{z}(t, r, z)\right)$ that is assumed to be parallel to the equilibrium magnetic field $\boldsymbol{B}=\left(B_{r}(r, z), 0,\left(B_{z}(r, z)\right)\right.$. The subscripts " $i$ " and " $e$ " indicate that a quantity is referred to inside the tube

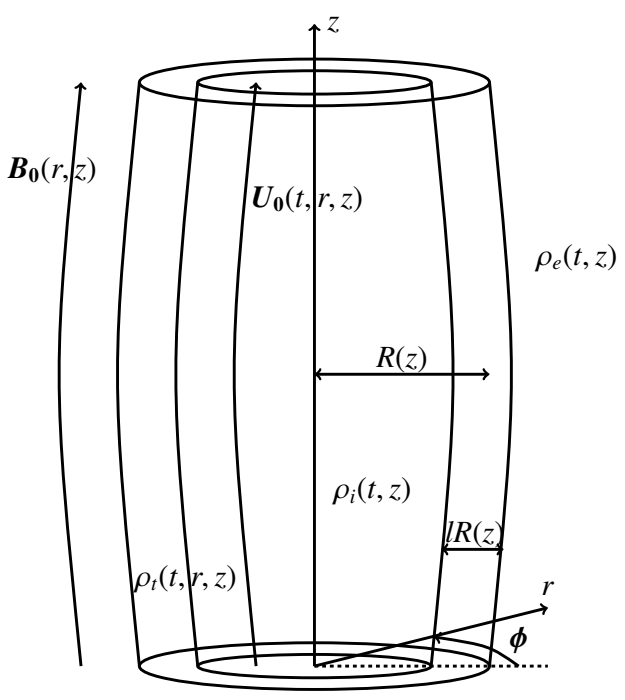

Fig. 1. Unperturbed configuration.

and in the external plasma, respectively. The plasma density and velocity are related by the mass conservation equation

$\frac{\partial \rho}{\partial t}+\frac{1}{r} \frac{\partial\left(r \rho U_{r}\right)}{\partial r}+\frac{\partial\left(\rho U_{z}\right)}{\partial z}=0$.

Ruderman et al. (2017) showed that in the thin tube approximation this equation reduces to the approximate form

$\frac{\partial \rho}{\partial t}+\frac{1}{R^{2}} \frac{\partial\left(\rho R^{2} U\right)}{\partial z}=0$

where $U \approx U_{z}$. They also obtained the approximate relation

$R^{2} B=$ const,

that follows from the divergent-free condition for the magnetic field. It follows from the divergence-free condition for the magnetic field that it can be expressed in terms of flux function $\psi$ as

$B_{r}=-\frac{1}{r} \frac{\partial \psi}{\partial z}, \quad B_{z}=\frac{1}{r} \frac{\partial \psi}{\partial r}$

The equations of the internal and external boundaries of the transitional region are $\psi=\psi_{\mathrm{i}}$ and $\psi=\psi_{\mathrm{e}}$, respectively. Given that we consider coronal environment, we use the cold plasma approximation. This is a viable assumption because a typical value of plasma beta is 0.01 in the solar corona. Then, it follows that, the equilibrium magnetic field must be potential. This condition results in the equation for $\psi$,

$r \frac{\partial}{\partial r}\left(\frac{1}{r} \frac{\partial \psi}{\partial r}\right)+\frac{\partial^{2} \psi}{\partial z^{2}}=0$

We introduce now the plasma displacement $\boldsymbol{\xi}=\left(\xi_{r}, \xi_{\phi}, \xi_{z}\right)$, the unit vector in the magnetic field direction $\boldsymbol{b}_{0}=\boldsymbol{B} / \boldsymbol{B}$, and the component of the displacement that is perpendicular to the magnetic field lines and in the $\phi=$ const planes,

$\xi_{\perp}=\xi_{r} b_{0 z}-\xi_{z} b_{0 r}$

Ruderman et al. (2017) showed that, in the thin tube and cold plasma approximation, kink oscillations of a flux tube with variable radius are described by the equation

$$
\begin{aligned}
& \rho_{\mathrm{i}}\left(\frac{\partial}{\partial t}+\frac{U_{\mathrm{i}}}{R^{2}} \frac{\partial}{\partial z} R^{2}\right)\left(\frac{\partial \eta}{\partial t}+U_{\mathrm{i}} \frac{\partial \eta}{\partial z}\right) \\
& \quad+\rho_{\mathrm{e}}\left(\frac{\partial}{\partial t}+\frac{U_{\mathrm{e}}}{R^{2}} \frac{\partial}{\partial z} R^{2}\right)\left(\frac{\partial \eta}{\partial t}+U_{\mathrm{e}} \frac{\partial \eta}{\partial z}\right)-\frac{2 B^{2}}{\mu_{0}} \frac{\partial^{2} \eta}{\partial z^{2}}=\mathcal{L}
\end{aligned}
$$


where

$$
\begin{aligned}
\mathcal{L} & =\frac{\delta P}{R^{2}}+\frac{B^{2}}{\mu_{0}} \frac{\partial^{2}(l \eta+\delta \eta)}{\partial z^{2}} \\
& -\rho_{\mathrm{e}}\left(\frac{\partial}{\partial t}+\frac{U_{\mathrm{e}}}{R^{2}} \frac{\partial}{\partial z} R^{2}\right)\left(\frac{\partial}{\partial t}+U_{\mathrm{e}} \frac{\partial}{\partial z}\right)(l \eta+\delta \eta),
\end{aligned}
$$

$U_{\mathrm{i}}$ and $U_{\mathrm{e}}$ are the flow velocities inside and outside the coronal loop respectively, $P$ is the magnetic pressure perturbation, $\mu_{0}$ is the magnetic permeability of free space, and

$\eta=\left.\frac{1}{R(z)} \xi_{\perp}\right|_{\psi=\psi_{\mathrm{i}}}$

$\delta P=\left.P\right|_{\psi=\psi_{\mathrm{e}}}-\left.P\right|_{\psi=\psi_{\mathrm{i}}}, \quad \delta \eta=\frac{1}{R(z)}\left(\left.\xi_{\perp}\right|_{\psi=\psi_{\mathrm{e}}}-\left.\xi_{\perp}\right|_{\psi=\psi_{\mathrm{i}}}\right)$

\section{Kink oscillations of coronal loops with slowly varying density}

\subsection{The WKB approximation}

In this section, we study kink oscillations of a magnetic flux tube with a variable cross-section and slowly varying density. We assume that the resonant damping is weak and the damping time is much larger than the oscillation period. Since the ratio of the oscillation period to the damping time is of the order of $l$ (see, e.g. Hollweg \& Yang 1988; Goossens et al. 1992; Ruderman \& Roberts 2002; Shukhobodskiy \& Ruderman 2018) this assumption is equivalent to the condition $l \ll 1$. We have already mentioned, Goossens et al. (2002) estimated the thickness of the transitional layer using 11 cases of observation of coronal kink oscillations. The largest value that they obtained was $l=0.49$, which does not look small. However, the numerical study by Van Doorsselaere et al. (2004a) showed that the thin boundary approximation gives fairly good approximation for the damping time for $l \lesssim 0.5$.

We aim to study the competition between the oscillation amplification due to cooling and damping due to resonant absorption. The oscillation amplification occurs on the timescale comparable to the characteristic time $t_{\mathrm{ch}}$ of the density variation (Ruderman 2011b; Ruderman et al. 2017), that is, in turn, of the order of the cooling time. Observations show that the typical cooling time is of the order as a few oscillation periods (Aschwanden \& Terradas 2008; Aschwanden \& Schrijver 2011). We would like to obtain the effects of damping and cooling in the same order approximation. Accordingly we formally assume that the characteristic cooling time is comparable to the damping time and take $t_{\mathrm{ch}}$ equal to $l^{-1}$ times the characteristic wave period. This assumption does not impose any restriction on the ratio of cooling and damping times. If the cooling time is, in fact, much larger than the damping time, then the effect of cooling can be neglected, and vise versa. In accordance with out assumption we introduce the "slow" time $t_{1}=l t$.

Now, we follow Ruderman (2011a) and use the WentzelKramers-Brillounin (WKB) method (see, e.g. Bender \& Orszag 1978) to seek the solution to the problem. In accordance with this method, we write

$\eta=S\left(t_{1}, z\right) \exp \left[i l^{-1} \Theta\left(t_{1}\right)\right]$.

Then we expand $S$ in the series

$S=S_{0}+l S_{1}+\ldots$
We have the estimate $\delta P \sim l P$. In accordance with these estimates, we now introduce the scaled jump of the magnetic pressure perturbation $\widetilde{\delta P}=l^{-1} \delta P$. We also have the estimate $\delta \eta \sim l \eta$. Since $\delta \eta=\delta S \mathrm{e}^{i l^{-1} \Theta}$, this estimate inspires us to introduce $\widetilde{\delta S}=l^{-1} \delta S$. Finally, it follows from Eq. (3) that $U \sim l$, so we introduce the scaled velocity $\widetilde{U}=l^{-1} U$. Now, substituting Eq. (12) in Eqs. (8) and (9) we obtain

$$
\begin{aligned}
& \left(\rho_{\mathrm{i}}+\rho_{\mathrm{e}}\right)\left[S \omega^{2}-i l\left(2 \omega \frac{\partial S}{\partial t_{1}}+S \frac{\partial \Omega}{\partial t_{1}}\right)\right] \\
& -\frac{2 i l \omega}{R}\left(\rho_{\mathrm{i}} \widetilde{U}_{\mathrm{i}}+\rho_{\mathrm{e}} \widetilde{U}_{\mathrm{e}}\right) \frac{\partial(R S)}{\partial z}+\frac{2 B^{2}}{\mu_{0}} \frac{\partial^{2} S}{\partial z^{2}}=-l \widetilde{\mathcal{L}}+O\left(l^{2}\right),
\end{aligned}
$$

$\widetilde{\mathcal{L}}=\left(\rho_{\mathrm{e}} \omega^{2}+\frac{B^{2}}{\mu_{0}} \frac{\partial^{2}}{\partial z^{2}}\right)(S+\widetilde{\delta S})+\frac{\widetilde{\delta P}}{R^{2}} \mathrm{e}^{-i l^{-1} \Theta}$,

where $\omega=\mathrm{d} \Theta / \mathrm{d} t_{1}$.

Substituting Eq. (13) in Eq. (14) and collecting terms of order of unity yields

$$
\frac{\partial^{2} S_{0}}{\partial z^{2}}+\frac{\omega^{2}}{C_{k}^{2}} S_{0}=0, \quad C_{k}^{2}=\frac{2 B^{2}}{\mu_{0}\left(\rho_{\mathrm{i}}+\rho_{\mathrm{e}}\right)} .
$$

This approximation is called the approximation of geometric optics. Since we assume that the tube ends are frozen in the dense photosphere, we impose the boundary conditions $\eta=0$ at $z= \pm L / 2$. Then, we obtain

$S_{0}=0 \quad$ at $\quad z= \pm L / 2$.

Equations (16) and (17) constitute the Sturm-Liouville problem for function $S_{0}$. This problem coincides with the boundary value problem obtained by Dymova \& Ruderman (2005) for kink oscillations of a magnetic tube with the density varying along the tube that is in a static equilibrium as well as with the boundary value problem obtained by Shukhobodskiy \& Ruderman (2018; hereafter Paper I) for kink oscillations of an expanding magnetic tube with the density varying along the tube that is in a static equilibrium. We assume that $\omega^{2}$ is the eigenvalue and $S_{0}$ is the corresponding eigenfunction. In accordance with the SturmLiouville theory, the eigenvalues are real and constitute a monotonically increasing sequence. Multiplying Eq. (17) by $S_{0}$ and integrating it over $z$ we have

$\omega^{2} \int_{-L / 2}^{L / 2} \frac{S_{0}^{2}}{C_{k}^{2}} \mathrm{~d} z=\int_{-L / 2}^{L / 2}\left(\frac{\partial S_{0}}{\partial z}\right)^{2} \mathrm{~d} z$.

This result implies that $\omega^{2}>0$.

Proceeding to the next order approximation, we collect terms of the order of $l$ in Eq. (14). This yields

$$
\begin{aligned}
\frac{\partial^{2} S_{1}}{\partial z^{2}}+\frac{\omega^{2}}{C_{k}^{2}} S_{1}= & \frac{i}{C_{k}^{2}}\left(2 \omega \frac{\partial S_{0}}{\partial t_{1}}+S_{0} \frac{\partial \omega}{\partial t_{1}}\right. \\
& \left.+\frac{2 \omega\left(\rho_{\mathrm{i}} \widetilde{U}_{\mathrm{i}}+\rho_{\mathrm{e}} \widetilde{U}_{\mathrm{e}}\right)}{R\left(\rho_{\mathrm{i}}+\rho_{\mathrm{e}}\right)} \frac{\partial\left(R S_{0}\right)}{\partial z}\right)-\frac{\widetilde{\mathcal{L}}}{C_{k}^{2}\left(\rho_{\mathrm{i}}+\rho_{\mathrm{e}}\right)}
\end{aligned}
$$

The function $S_{1}$ must satisfy the boundary conditions

$S_{1}=0 \quad$ at $\quad z= \pm L / 2$.

The homogeneous counterpart of Eq. (19) with the boundary conditions given by Eq. (20) has a non-trivial solution $S_{1}=S_{0}$. 
This implies that the boundary value problem determining $S_{1}$ has solutions only if the right-hand side of Eq. (19) satisfies the compatibility condition, which is the condition that it must be orthogonal to $S_{0}$. To obtain this condition, we multiply Eq. (19) by $S_{0}$, integrate the obtained equation with respect to $z$, and use the integration by parts and the boundary conditions given by Eqs. (17) and (20). As a result, we obtain

$$
\begin{aligned}
\int_{-L / 2}^{L / 2}\left(\frac{\partial\left(\omega S_{0}^{2}\right)}{\partial t_{1}}\right. & \left.+\frac{\omega\left(\rho_{\mathrm{i}} \widetilde{U}_{\mathrm{i}}+\rho_{\mathrm{e}} \widetilde{U}_{\mathrm{e}}\right)}{R^{2}\left(\rho_{\mathrm{i}}+\rho_{\mathrm{e}}\right)} \frac{\partial\left(R^{2} S_{0}^{2}\right)}{\partial z}\right) \frac{\mathrm{d} z}{C_{k}^{2}} \\
& =-i \int_{-L / 2}^{L / 2} \frac{\widetilde{\mathcal{L}} \mathrm{d} z}{C_{k}^{2}\left(\rho_{\mathrm{i}}+\rho_{\mathrm{e}}\right)} .
\end{aligned}
$$

Using integration by parts and Eqs. (3), (4) and (17) we obtain the identity

$$
\begin{aligned}
& \int_{-L / 2}^{L / 2} \frac{\left(\rho_{\mathrm{i}} \widetilde{U}_{\mathrm{i}}+\rho_{\mathrm{e}} \widetilde{U}_{\mathrm{e}}\right)}{R^{2} C_{k}^{2}\left(\rho_{\mathrm{i}}+\rho_{\mathrm{e}}\right)} \frac{\partial\left(R^{2} S_{0}^{2}\right)}{\partial z} \mathrm{~d} z \\
& =\frac{\mu_{0}}{2 R^{4} B^{2}} \int_{-L / 2}^{L / 2} R^{2}\left(\rho_{\mathrm{i}} \widetilde{U}_{\mathrm{i}}+\rho_{\mathrm{e}} \widetilde{U}_{\mathrm{e}}\right) \frac{\partial\left(R^{2} S_{0}^{2}\right)}{\partial z} \mathrm{~d} z \\
& =-\frac{\mu_{0}}{2} \int_{-L / 2}^{L / 2} \frac{S_{0}^{2}}{R^{2} B^{2}} \frac{\partial}{\partial z}\left[R^{2}\left(\rho_{\mathrm{i}} \widetilde{U}_{\mathrm{i}}+\rho_{\mathrm{e}} \widetilde{U}_{\mathrm{e}}\right)\right] \mathrm{d} z \\
& =\frac{\mu_{0}}{2} \int_{-L / 2}^{L / 2} \frac{S_{0}^{2}}{B^{2}} \frac{\partial\left(\rho_{\mathrm{i}}+\rho_{\mathrm{e}}\right)}{\partial t_{1}} \mathrm{~d} z=\int_{-L / 2}^{L / 2} S_{0}^{2} \frac{\partial C_{k}^{-2}}{\partial t_{1}} \mathrm{~d} z .
\end{aligned}
$$

Using this identity and returning to the non-scaled time we transform Eq. (21) to

$$
\frac{\mathrm{d}}{\mathrm{d} t}\left(\omega \int_{-L / 2}^{L / 2} \frac{S_{0}^{2}}{C_{k}^{2}} \mathrm{~d} z\right)=-i l \int_{-L / 2}^{L / 2} \frac{S_{0} \widetilde{\mathcal{L}} \mathrm{d} z}{C_{k}^{2}\left(\rho_{\mathrm{i}}+\rho_{\mathrm{e}}\right)} .
$$

The quantity in the brackets on the left-hand side of this equation is called adiabatic invariant. It can be interpreted as the wave action. When $l=0$ the right-hand side is zero and the adiabatic invariant is conserved. This result was previously obtained by Ruderman et al. (2017). It is worth noting that the oscillation energy is not conserved because, in general, there is the plasma flow through the loop footpoints. Even if we impose the condition that there is no plasma flow through the footpoints, there is the plasma redistribution in the loop caused by cooling. As a result, there is the exchange of energy between the oscillation and gravity field.

\subsection{Calculation of $\widetilde{\mathcal{L}}$}

Equation (23) describes the evolution of $S_{0}$ and, consequently, the oscillation amplitude. This equation is not closed because the expression for $\widetilde{\mathcal{L}}$ contains $\widetilde{\delta S}$ and $\widetilde{\delta P}$. To close it, we need to express $\widetilde{\delta S}$ and $\widetilde{\delta P}$ in terms of $S_{0}$. Since we only need to calculate the right-hand side of Eq. (23) in the leading order approximation with respect to $l$, it follows that we need to calculate $\widetilde{\delta S}$ and $\widetilde{\delta P}$ also only in the leading order approximation. Using Eq. (3), we obtain the estimate that $U_{\mathrm{i}, \mathrm{e}} \sim l C_{k}$. It also follows that $\partial S_{0} / \partial t \sim l \omega S_{0}$. These estimates imply that the account of the flow in the transitional layer and the time derivative of $S$ can only give corrections of order $l$ to $\widetilde{\delta S}$ and $\widetilde{\delta P}$. Hence, we can neglect the flow and the time derivative of $S$ when calculating these quantities. Then, we can use the same equations as in Paper I to calculate $\widetilde{\delta S}$ and $\widetilde{\delta P}$. However, we cannot directly use the results obtained in Paper I. The reason is that it was assumed in Paper I that the unperturbed density is equal to the product of two functions, one depending on $\psi$, and the other on $z$. Here, we cannot make this assumption because even if it is satisfied at the initial time, in general, later it will be not valid because of the density variation with time. Hence, we need to modify the analysis given by Paper I. Below, we briefly describe this analysis and the modification that we make.

Following Paper I, let us use the variable $\psi$ instead of $r$. Then, we consider Alfvén oscillations of individual magnetic filed lines, which are described by the eigenvalue problem

$V_{\mathrm{A}}^{2} \frac{\partial^{2} Y}{\partial z^{2}}=-\lambda Y, \quad Y=0$ at $z= \pm L / 2$,

where

$V_{\mathrm{A}}^{2}=\frac{B^{2}}{\mu_{0} \rho}$

is the Alfvén speed. Here, $Y$ and $V_{\mathrm{A}}$ depend on $t, \psi$, and $z$, and $\lambda$ on $t$ and $\psi$. The eigenvalues of this problem are real and constitute a monotonically increasing sequence $\lambda_{n}$, where $\lambda_{n} \rightarrow \infty$ as $n \rightarrow \infty$ (e.g. Coddington \& Levinson 1955). It is easy to show that all eigenvalues are positive. Any square integrable function $f(z)$ in the interval $[-L / 2, L / 2]$ can be expanded in a generalised Fourier series

$f(t, \psi, z)=\sum_{n=1}^{\infty} f_{n}(t, \psi) Y_{n}(t, \psi, z)$,

where $Y_{n}(t, \psi, z)$ is an eigenfunction of the boundary value problem (24). Obviously, all $Y_{n}$ can be chosen to be real. According to the classical Sturm-Liouville theory the eigenfunctions corresponding to different eigenvalues are orthogonal with the weigth $V_{\mathrm{A}}^{-2}(t, \psi, z)$. In addition, we can normalise them in such a way that they satisfy the relation

$\int_{-L / 2}^{L / 2} V_{\mathrm{A}}^{-2}(z) Y_{m}(z) Y_{n}(z) \mathrm{d} z=\delta_{m n}$,

where $\delta_{m n}$ is the Kronecker delta-symbol. If $f(z)$ has a continuous second derivative and satisfies the boundary condition $f( \pm L / 2)=0$, then the sum in (26) is uniformly convergent and can be differentiated twice (see, e.g. Naimark 1967). The Fourier coefficients in the Fourier series (26) are given by

$f_{n}=\int_{-L / 2}^{L / 2} V_{\mathrm{A}}^{-2}(z) f(z) Y_{n}(z) \mathrm{d} z$.

The resonance of a global kink oscillation with the $n$th harmonic of local Alfvén oscillations occurs at the resonant magnetic surface defined by the equation $\psi=\psi_{n}$ if the relation $\lambda_{n}\left(\psi_{n}\right)=\omega^{2}$ is satisfied. Since $\lambda_{n} \rightarrow \infty$ as $n \rightarrow \infty$, it follows that only a finite number of Alfvén resonances exist.

Observations show that, in most cases, the fundamental harmonic of kink oscillations is dominant, so the oscillation amplitude is determined by the fundamental harmonic. In accordance with this, in what follows, we restrict our analysis to the fundamental harmonic. We assume that $\rho(t, \psi, z)$ is a monotonically decreasing function of $\psi$ for all $z \in[-L / 2, L / 2]$ and at any time. Then $V_{\mathrm{Ai}}(z)<C_{k}(z)<V_{\mathrm{Ae}}(z)$ for all $z \in[-L / 2, L / 2]$. From using the comparison theorem for ordinary differential equations (e.g. Coddington \& Levinson 1955), it is straightforward to show that $\lambda_{1}\left(\psi_{\mathrm{i}}\right)<\omega^{2}<\lambda_{1}\left(\psi_{\mathrm{e}}\right)$. This implies that there is $\psi_{1} \in\left[\psi_{\mathrm{i}}, \psi_{\mathrm{e}}\right]$ such that $\lambda_{1}\left(\psi_{1}\right)=\omega^{2}$. Hence, there is always at least one resonant surface in the transitional layer. Below, we 
assume that $\lambda_{2}\left(\psi_{\mathrm{i}}\right)>\lambda_{1}\left(\psi_{\mathrm{e}}\right)$. Then, it follows that there is exactly one resonant surface.

Now, the derivation of expressions for $\delta P$ and $\delta \eta$ almost completely repeats the derivation of expressions for these quantities given in Paper I. The only difference is the following. In accordance with the assumption about the density made in Paper I, $V_{\mathrm{A}}^{2}=V_{\mathrm{Ai}}^{2} g(\psi)$, where $g(\psi)$ is a monotonically increasing function and $g\left(\psi_{\mathrm{i}}\right)=1$. Then, in Paper I, the following expansion is used:

$\frac{V_{\mathrm{Ai}}^{2} Q_{\mathrm{i}}}{R^{2}}=\sum_{n=1}^{\infty} \Phi_{n} Y_{n}(z)$

where $Q=P / B^{2}$. Since in this paper $V_{\mathrm{A}}^{2}$ cannot be factorised, we use instead the expansion

$\frac{V_{\mathrm{A}}^{2} Q}{R^{2}}=\sum_{n=1}^{\infty} \Phi_{n}(\psi) Y_{n}(\psi, z)$.

After that we obtain the expressions for $\delta Q$ and $\delta \eta$ substituting $\Phi_{n}(\psi)$ for $\Phi_{n} g(\psi)$ in all Eqs. from (41) to (63) in Paper I. They read

$$
\begin{aligned}
\delta Q & =\int_{\psi_{\mathrm{i}}}^{\psi_{\mathrm{e}}}\left(\frac{Q_{\mathrm{i}}}{\psi_{\mathrm{i}}}-\frac{\left(\rho_{\mathrm{i}}-\rho\right) \omega^{2} \eta}{B^{3}}\right) \mathrm{d} \psi, \\
\delta \eta & =\frac{\mu_{0}}{B R^{2}} \mathcal{P} \int_{\psi_{\mathrm{i}}}^{\psi_{\mathrm{e}}} \sum_{n=1}^{\infty} \frac{\Phi_{n}(t, \psi) Y_{n}(t, \psi, z)}{\omega^{2}(t)-\lambda_{n}(t, \psi)} \mathrm{d} \psi \\
& -\frac{\pi i \mu_{0} \Phi_{1}\left(t, \psi_{1}\right) Y_{1}\left(t, \psi_{1}, z\right)}{|\Delta(t)| B R^{2}},
\end{aligned}
$$

where $\mathcal{P}$ indicates the principal Cauchy part of the integral and

$\Delta=-\left.\frac{\mathrm{d} \lambda_{1}}{\mathrm{~d} \psi}\right|_{\psi=\psi_{1}}$.

When deriving Eq. (32) we took into account that $B R^{2}=$ const. Now, we use the Eq. (30) from Paper I where we substitute $\omega$ for $\Omega, v$ for $\bar{v}$, and $z$ for $Z$ because, in this article, we do not use the scaled frequency, kinematic viscosity, and the coordinate along the tube. Then, we obtain

$V_{\mathrm{A}}^{2} \frac{\partial^{2} W}{\partial z^{2}}+\omega^{2} W-i v \omega R^{2} B^{2} \frac{\partial^{2} W}{\partial \psi^{2}}=\frac{\mu_{0} V_{\mathrm{A}}^{2} Q_{\mathrm{i}}}{R^{2}}$,

where

$W=\frac{\partial\left(r B \xi_{\perp}\right)}{\partial \psi}$.

The last term on the left-hand side of Eq. (34) describing the effect of viscosity is only important in the thin dissipative layer embracing the resonant surface. Below, we use Eq. (34) outside of the dissipative layer and thus can neglect this term. Ruderman et al. (2017) showed that in the thin tube approximation $B$ and $\xi_{\perp}$ are independent of $\psi$ and $\psi=\frac{1}{2} r^{2} B$. Then, using the approximation $r \approx R$ valid in the transitional layer we obtain $W=\eta$. As a result, Eq. (34) reduces to

$V_{\mathrm{A}}^{2} \frac{\partial^{2} \eta}{\partial z^{2}}+\omega^{2} \eta=\frac{\mu_{0} V_{\mathrm{A}}^{2} Q_{\mathrm{i}}}{R^{2}}$

We take this equation at $\psi=\psi_{\mathrm{i}}$. Then, using Eq. (16) we obtain in the leading order approximation with respect to $l$

$Q_{\mathrm{i}}=\frac{\omega^{2} R^{2} S_{0}\left(\rho_{\mathrm{i}}-\rho_{\mathrm{e}}\right)}{2 B^{2}} \mathrm{e}^{i l^{-1} \Theta}$.
Substituting this result in Eq. (31), noticing that $\psi_{\mathrm{i}}=\frac{1}{2} B R^{2}[1+$ $O(l)]$, and using the relation $\widetilde{\delta P}=l^{-1} B^{2} \delta Q$ yields

$\widetilde{\delta P}=\frac{\omega^{2} l^{-1} S_{0}}{B} \mathrm{e}^{i l^{-1} \Theta} \int_{\psi_{\mathrm{i}}}^{\psi_{\mathrm{e}}}\left(\rho-\rho_{\mathrm{e}}\right) \mathrm{d} \psi$.

Now, we need to express $\widetilde{\delta S}$ in terms of $S_{0}$. We have $\widetilde{\delta S}=$ $l^{-1} \delta \eta \mathrm{e}^{-i l^{-1} \Theta}$, where $\delta \eta$ is given by Eq. (32). This equation contains the functions $\Phi_{n}(t, \psi)$, so we need to express these functions in terms of $S_{0}$. To do this, we use Eq. (30). Since we only need to calculate $\Phi_{n}(t, \psi)$ in the leading order approximation with respect to $l$, we can substitute $Q_{\mathrm{i}}$ for $Q$ in this equation. Then, using Eqs. (28) and (37) we obtain

$\Phi_{n}=\omega^{2} \mathrm{e}^{i l^{-1} \Theta} \int_{-L / 2}^{L / 2} \frac{S_{0} Y_{n}\left(\rho_{\mathrm{i}}-\rho_{\mathrm{e}}\right)}{2 B^{2}} \mathrm{~d} z$.

Substituting this expression in Eq. (32) and using the relation between $\widetilde{\delta S}$ and $\delta \eta$ yields

$$
\begin{aligned}
\widetilde{\delta S}= & \frac{l^{-1} \pi i \mu_{0} \omega^{2} Y_{1}\left(\psi_{1}, z\right)}{|\Delta| B R^{2}} \int_{-L / 2}^{L / 2} \frac{S_{0}(\tilde{z}) Y_{1}\left(\psi_{1}, \tilde{z}\right)\left[\rho_{\mathrm{e}}(\tilde{z})-\rho_{\mathrm{i}}(\tilde{z})\right]}{2 B^{2}(\tilde{z})} \mathrm{d} \tilde{z} \\
& +\frac{l^{-1} \mu_{0} \omega^{2}}{B R^{2}} \mathcal{P} \int_{\psi_{\mathrm{i}}}^{\psi_{\mathrm{e}}}\left(\sum_{n=1}^{\infty} \frac{Y_{n}(z)}{\omega^{2}-\lambda_{n}}\right. \\
& \left.\times \int_{-L / 2}^{L / 2} \frac{S_{0}(\tilde{z}) Y_{n}(\tilde{z})\left[\rho_{\mathrm{i}}(\tilde{z})-\rho_{\mathrm{e}}(\tilde{z})\right]}{2 B^{2}(\tilde{z})} \mathrm{d} \tilde{z}\right) \mathrm{d} \psi
\end{aligned}
$$

where we only showed the dependence on $z$, but we did not show the dependence on $t$ and $\psi$. Finally, using Eqs. (15), (16), (38) and (40) we obtain

$$
\begin{aligned}
\widetilde{\mathcal{L}} & =l^{-1} \omega^{2} S_{0}(z)\left(\int_{\psi_{\mathrm{i}}}^{\psi_{\mathrm{e}}} \frac{\rho(z)-\rho_{\mathrm{i}}(z)}{B(z) R^{2}(z)} \mathrm{d} \psi-\frac{\rho_{\mathrm{i}}(z)-\rho_{\mathrm{e}}(z)}{2}\right) \\
& +\frac{\mu_{0} \omega^{2}}{B R^{2}} \mathcal{P} \int_{\psi_{\mathrm{i}}}^{\psi_{\mathrm{e}}}\left(\sum_{n=1}^{\infty} \frac{Y_{n}(z)\left[\omega^{2} \rho_{\mathrm{e}}(z)-\lambda_{n} \rho(z)\right]}{\omega^{2}-\lambda_{n}}\right. \\
& \left.\times \int_{-L / 2}^{L / 2} \frac{S_{0}(\tilde{z}) Y_{n}(\tilde{z})\left[\rho_{\mathrm{i}}(\tilde{z})-\rho_{\mathrm{e}}(\tilde{z})\right]}{2 B^{2}(\tilde{z})} \mathrm{d} \tilde{z}\right) \mathrm{d} \psi \\
& +\frac{l^{-1} \pi i \mu_{0} \omega^{4} Y_{1}\left(\psi_{1}, z\right)\left[\rho\left(\psi_{1}, z\right)-\rho_{\mathrm{e}}(z)\right]}{|\Delta| B R^{2}} \\
& \times \int_{-L / 2}^{L / 2} \frac{S_{0}(\tilde{z}) Y_{1}\left(\psi_{1}, \tilde{z}\right)\left[\rho_{\mathrm{i}}(\tilde{z})-\rho_{\mathrm{e}}(\tilde{z})\right]}{2 B^{2}(\tilde{z})} \mathrm{d} \tilde{z}
\end{aligned}
$$

\subsection{Amplitude variation}

We consider an eigenfunction $X(t, z)$ of the boundary value problem constituted by Eqs. (16) and (17) that is real-valued, corresponds to the fundamental mode, and satisfies the condition $\max _{z} X(t, z)=1$. Since $S_{0}$ is also an eigenvalue of the same boundary value problem it must be proportional to $X(t, z)$ with the proportionality coefficient depending on time. Hence, we can write

$S_{0}(t, z)=A(t) \mathrm{e}^{i F(t)} X(t, z)$,

where $A(t)$ and $F(t)$ are real-valued functions, and $A(t)>0$. Since $\max _{z}|S(t, z)|=A(t)$, the function $A(t)$ can be considered as the oscillation amplitude. Using Eq. (42) we obtain

$$
\begin{aligned}
\frac{\mathrm{d}}{\mathrm{d} t}\left(\omega \int_{-L / 2}^{L / 2} \frac{S_{0}^{2}}{C_{k}^{2}} \mathrm{~d} z\right)= & \mathrm{e}^{2 i F(t)} \frac{\mathrm{d}}{\mathrm{d} t}\left(\omega A^{2} \int_{-L / 2}^{L / 2} \frac{X^{2}}{C_{k}^{2}} \mathrm{~d} z\right) \\
& +2 i \omega A^{2} \mathrm{e}^{2 i F(t)} \frac{\mathrm{d} F}{\mathrm{~d} t} \int_{-L / 2}^{L / 2} \frac{X^{2}}{C_{k}^{2}} \mathrm{~d} z .
\end{aligned}
$$


With the aid of Eq. (41) we calculate the right-hand side of Eq. (23),

$-i l \int_{-L / 2}^{L / 2} \frac{S_{0} \widetilde{\mathcal{L}} \mathrm{d} z}{C_{k}^{2}\left(\rho_{\mathrm{i}}+\rho_{\mathrm{e}}\right)}=A^{2} \mathrm{e}^{2 i F(t)}(\Gamma+i \omega \Upsilon)$,

where

$$
\begin{aligned}
\Gamma= & \frac{\pi \mu_{0}^{2} \omega^{4}}{|\Delta| B R^{2}} \int_{-L / 2}^{L / 2} \frac{X Y_{1}\left(\psi_{1}\right)}{2 B^{2}}\left[\rho\left(\psi_{1}\right)-\rho_{\mathrm{e}}\right] \mathrm{d} z \\
& \times \int_{-L / 2}^{L / 2} \frac{X Y_{1}\left(\psi_{1}\right)\left(\rho_{\mathrm{i}}-\rho_{\mathrm{e}}\right)}{2 B^{2}} \mathrm{~d} z \\
\Upsilon & =\omega \mathrm{e}^{2 i F(t)}\left[\int_{-L / 2}^{L / 2} \frac{X^{2}}{2 C_{k}^{2}}\left(\frac{\rho_{\mathrm{i}}-\rho_{\mathrm{e}}}{\rho_{\mathrm{i}}+\rho_{\mathrm{e}}}-2 \int_{\psi_{\mathrm{i}}}^{\psi_{\mathrm{e}}} \frac{\left(\rho-\rho_{\mathrm{i}}\right) \mathrm{d} \psi}{B R^{2}\left(\rho_{\mathrm{i}}+\rho_{\mathrm{e}}\right)}\right) \mathrm{d} z\right. \\
& -\frac{\mu_{0}}{B R^{2}} \mathcal{P} \int_{\psi_{\mathrm{i}}}^{\psi_{\mathrm{e}}}\left(\sum_{n=1}^{\infty} \int_{-L / 2}^{L / 2} \frac{X Y_{n}\left(\omega^{2} \rho_{\mathrm{e}}-\lambda_{n} \rho\right)}{C_{k}^{2}\left(\rho_{\mathrm{i}}+\rho_{\mathrm{e}}\right)\left(\omega^{2}-\lambda_{n}\right)}\right. \\
& \left.\left.\times \int_{-L / 2}^{L / 2} \frac{X Y_{n}\left(\rho_{\mathrm{i}}-\rho_{\mathrm{e}}\right)}{2 B^{2}} \mathrm{~d} z\right) \mathrm{~d} \psi\right] .
\end{aligned}
$$

Substituting Eqs. (43) and (44) in Eq. (23) we eventually arrive at

$\frac{\mathrm{d}}{\mathrm{d} t}\left(\omega A^{2} \int_{-L / 2}^{L / 2} \frac{X^{2}}{C_{k}^{2}} \mathrm{~d} z\right)=-\Gamma A^{2}$,

$\frac{\mathrm{d} F}{\mathrm{~d} t} \int_{-L / 2}^{L / 2} \frac{X^{2}}{C_{k}^{2}} \mathrm{~d} z=\Upsilon$.

Equation (47) describes the evolution of the oscillation amplitude with time. The function $F(t)$ determines the phase shift related to the presence of the transitional layer.

\section{Kink oscillations of coronal loops with barometric density distribution}

\subsection{Kink oscillations of static coronal loops}

We, now, verify that Eq. (47) correctly describes the damping of kink oscillations of static coronal loops. In the case of a static loop, Eq. (47) becomes

$\frac{\mathrm{d} A}{\mathrm{~d} t}=\gamma A, \quad \gamma=\frac{\Gamma}{2 \omega I}$,

where

$I=\int_{-L / 2}^{L / 2} \frac{X^{2}}{C_{k}^{2}} \mathrm{~d} z$.

It follows form Eq. (49) that oscillation amplitude decreases exponentially with the decrement $\gamma$. Let $\zeta=\rho_{\mathrm{i}}(L / 2) / \rho_{\mathrm{e}}(L / 2)$ Similarly to Paper I, we assume, that $\rho_{\mathrm{i}}(z) / \rho_{\mathrm{e}}(z)=\zeta$ and $\rho_{t}(\psi, z)=\rho_{\mathrm{i}}(z) / g(\psi)$, where $g(\psi)$ is a monotonically increasing function satisfying $g\left(\psi_{\mathrm{i}}\right)=1$ and $g\left(\psi_{\mathrm{e}}\right)=V_{\mathrm{Ae}}^{2} / V_{\mathrm{Ai}}^{2}$. As a result, following the analysis in Paper I, we can write $\lambda_{1}$ as

$\lambda_{1}(\psi)=\lambda_{1}\left(\psi_{\mathrm{i}}\right) g(\psi)$.

We recall our assumption that there is only one resonant surface $\psi=\psi_{1}$. Now, we can rewrite Eq. (16) as

$\frac{B^{2}}{\mu_{0} \rho_{\mathrm{e}}(z)} \frac{\partial^{2} S_{0}}{\partial z^{2}}=-\frac{\omega^{2}(\zeta+1)}{2} S_{0}$
For fundamental mode at $\psi=\psi_{1}$ Eq. (24) can be rewritten as

$\frac{B^{2}}{\mu_{0} \rho_{\mathrm{e}}(z)} \frac{\partial^{2} Y_{1}\left(\psi_{1}\right)}{\partial z^{2}}=-\frac{\zeta \lambda_{1}\left(\psi_{1}\right)}{g\left(\psi_{1}\right)} Y_{1}\left(\psi_{1}\right)$.

We see that both $S_{0}$ and $Y_{1}$ are eigenfunctions of the same differential operator corresponding to the fundamental mode. This implies that the coefficients on the right-hand sides of Eqs. (52) and (53) must be equal. Then, it follows from the resonant condition, $\lambda_{1}=\omega^{2}$, that

$g\left(\psi_{1}\right)=\frac{2 \zeta}{\zeta+1}$.

Substituting Eqs. (51) and (54) in Eq. (33) yields

$\Delta=-\omega^{2} g^{\prime}\left(\psi_{1}\right)$.

Using Eq. (54) we obtain

$\rho_{\mathrm{e}}(z)=\frac{2 \rho\left(\psi_{1}\right)}{\zeta+1}$.

The functions $X(z)$ and $Y_{1}\left(\psi_{1}, z\right)$ are the eigenfunctions of the same eigenvalue problem corresponding to the same eigenvalue. Therefore, we have $X=\varsigma Y_{1}\left(\psi_{1}\right)$, where $\varsigma$ is a constant. Using this result and Eqs. (27), (55) and (56) we transform Eq. (45) to

$\Gamma=\frac{\pi \varsigma^{2} \omega^{2} \zeta(\zeta-1)^{2}}{B R^{2} g^{\prime}\left(\psi_{1}\right)(\zeta+1)^{3}}$.

Again, using the relation $X=\varsigma Y_{1}\left(\psi_{1}\right)$, and also Eqs. (27) and (56) we obtain $I=\varsigma^{2}$. Using this result and Eq. (57) yields

$\gamma=\frac{\pi \omega \zeta(\zeta-1)^{2}}{2 B R^{2} g^{\prime}\left(\psi_{1}\right)(\zeta+1)^{3}}$.

Following the analysis in Paper I, we consider the linear density profile in the transitional layer and take

$\rho_{t}(r, z)=\frac{\rho_{\mathrm{i}}+\rho_{\mathrm{e}}}{2}+\left(\rho_{\mathrm{i}}-\rho_{\mathrm{e}}\right) \frac{R-r}{l R}$,

Then, using Eq. (54) and the relation $\psi=\frac{1}{2} B r^{2}$ we obtain in the leading order approximation

$g^{\prime}\left(\psi_{1}\right)=\frac{4 \zeta(\zeta-1)}{l B R^{2}(\zeta+1)^{2}}$.

Substituting this expression in Eq. (58) yields

$\frac{\gamma}{\omega}=\frac{\pi l(\zeta-1)}{8(\zeta+1)}$

This expression coincides with that obtained by Goossens et al. (2002) for a tube with the density not varying along the tube and in Paper I.

Next, we consider sinusoidal profile determined by

$\rho_{t}(r, z)=\frac{\rho_{\mathrm{i}}+\rho_{\mathrm{e}}}{2}-\frac{\rho_{\mathrm{i}}-\rho_{\mathrm{e}}}{2} \sin \left(\frac{\pi(r-R)}{l R}\right)$.

Using the relation $\psi=\frac{1}{2} B r^{2}$ we obtain

$r=R\left(1-\frac{l}{2}\right)+\frac{\psi-\psi_{\mathrm{i}}}{B R}+O\left(l^{2}\right)$.

A173, page 6 of 10 
It follows from Eqs. (62) and (63) that

$$
\frac{1}{g(\psi)}=\frac{\zeta+1}{2 \zeta}+\frac{\zeta-1}{2 \zeta} \cos \frac{\pi\left(\psi-\psi_{\mathrm{i}}\right)}{l B R^{2}} \text {. }
$$

With the aid of Eqs. (54) and (64) we obtain

$\psi_{1}=\psi_{\mathrm{i}}+\frac{1}{2} l B R^{2}$

Then, using Eqs. (54), (64) and (65) yields

$g^{\prime}\left(\psi_{1}\right)=\frac{2 \pi \zeta(\zeta-1)}{l B R^{2}(\zeta+1)^{2}}$.

Substituting this result in Eq. (58) we arrive at

$\frac{\gamma}{\omega}=\frac{l(\zeta-1)}{4(\zeta+1)}$

This expression coincides with that obtained by Ruderman \& Roberts (2002) who considered damping of kink oscillations of a magnetic tube with the constant crosssection and density not varying along the tube. This result again confirms the conclusion made in Paper I that the ratio $\gamma / \omega$ is not affected by the density and cross-section radius variation along the tube if $\rho_{t}(\psi, z)=\rho_{\mathrm{i}}(z) / g(\psi)$.

\subsection{Kink oscillations of cooling coronal loops}

In this section, we again assume that there is only one resonant position $\psi=\psi_{1}$. Also, similarly to Ruderman (2011a) we assume that the temperature of plasma outside the loop does not change with time and is $T_{0}$, while inside the loop it decreases with time due to the effect of radiative cooling. The loop cooling occurs due to radiation. The radiation energy flux is proportional to the density square. Since the density in the loop is substantially higher than the density of the surrounded plasma the assumption that cooling only occurs in the tube looks like a viable assumption. Similar to Aschwanden \& Terradas (2008), Morton \& Erdélyi (2010), Ruderman (2011b) and Ruderman et al. (2017) we approximate the temperature evolution inside the loop by the exponentially decaying function,

$T(t)=T_{0} \exp \left(-t / t_{\mathrm{cool}}\right)$

where $t_{\text {cool }}$ is the cooling time. Following Ruderman et al. (2008, 2017) we describe the variation of the loop cross-section as

$R(z)=R_{f} \vartheta \sqrt{\frac{\cosh \left(L / 2 L_{c}\right)-1}{\cosh \left(L / 2 L_{c}\right)-\vartheta^{2}+\left(\vartheta^{2}-1\right) \cosh \left(z / L_{c}\right)}}$,

where $R_{f}$ is the cross-section radius at the footpoints, $L_{c}$ is and arbitrary positive constant with the dimension of length, and $\vartheta=$ $R(0) / R_{f}$ is the expansion factor. Ruderman et al. (2008) showed that the $z$-component of magnetic field is positive in everywhere in the region $|z| \leq L / 2$ only if $\vartheta<\vartheta_{m}$, where

$\vartheta_{m}^{2} \approx \frac{1.4 \cosh \left(L / 2 L_{c}\right)}{1+0.4 \cosh \left(L / 2 L_{c}\right)}$.

It follows from this equation that $\vartheta_{m}$ is a monotonically increasing fucntion of $L / L_{c}, \vartheta_{m} \rightarrow 1$ as $L / L_{c} \rightarrow 0$, and $\vartheta_{m} \rightarrow 1.87$ as $L / L_{c} \rightarrow \infty$. Typical coronal loop expansion does not exceed 1.5, thus by varying $L / L_{c}$ we can cover the whole range of values of the expansion factor. In what follows, we consider a loop with a half-circle shape. We neglect the effect of the loop curvature on oscillations because, as it was shown by Van Doorsselaere et al. (2004b) and Terradas et al. (2006), it is very weak. As a result, the loop shape only determines the density variation along the loop. The density outside the loop is given by the barometric formula,

$\rho_{\mathrm{e}}(z)=\frac{\rho_{f}}{\zeta} \exp \left(-\frac{L}{\pi H_{0}} \cos \frac{\pi z}{L}\right)$,

where $\rho_{f}$ is the plasma density at the footpoints inside the loop at $t=0, \zeta$ is the ratio of densities inside and outside the loop at the footpoints and at $t=0$,

$H_{0}=\frac{k_{\mathrm{B}} T_{0}}{m g}$

$k_{\mathrm{B}}$ is the Boltzmann constant, $m$ is the mean mass per particle approximately equal to $0.6 m_{\mathrm{p}}$ in the solar corona, $m_{\mathrm{p}}$ is the proton mass, and $g$ is the gravity acceleration. Ruderman (2011a) showed that under typical coronal conditions and observed cooling time, the effect of flow inside the coronal loop due to cooling is fairly weak. That justifies the use of barometric approximation inside a cooling coronal loop. As a result, we have

$\rho_{\mathrm{i}}(z)=\rho_{f} \exp \left(-\frac{L}{\pi H(t)} \cos \frac{\pi z}{L}\right)$

where

$H(t)=\frac{k_{\mathrm{B}} T(t)}{m g}$.

We also assume that the density profile in the transitional layer is linear, therefore

$\rho_{t}(t, r, z)=\frac{1}{2}\left[\rho_{\mathrm{i}}(t, z)+\rho_{\mathrm{e}}(z)\right]+\left[\rho_{\mathrm{i}}(t, z)-\rho_{\mathrm{e}}(z)\right] \frac{R-r}{l R}$.

It follows that $\rho_{t}=\left(\rho_{\mathrm{i}}+\rho_{\mathrm{e}}\right) / 2$ at $r=R$. It is straightforward to see that $V_{\mathrm{A}}=C_{k}$ when $r=R$, the boundary value problems for $S_{0}$ and $Y$ are the same and, consequently, $\lambda_{1}=\omega^{2}$ and $r=R$ is the resonant surface. Then, using the relation $\psi=\frac{1}{2} B r^{2}$, we obtain that $\psi_{1}=\frac{1}{2} B R^{2}$.

The functions $X$ and $Y_{1}\left(\psi_{1}\right)$ are defined by the same boundary value problem which implies that $Y_{1}\left(\psi_{1}\right)$ is proportional to $X$ and $Y_{1}\left(\psi_{1}\right)$. Since the equilibrium is symmetric with respect to the apex point, it follows that $X(z)$ describing the fundamental mode is an even function. Hence, it takes maximum at $z=0$, and thus the condition $\max _{z}(X)=1$ reduces to $X(0)=1$. Summarising, we obtain

$Y_{1}\left(\psi_{1}, z\right)=Y_{1}\left(\psi_{1}, 0\right) X(z)$.

Then it follows from Eq. (27) and the relation $V_{\mathrm{A}}\left(\psi_{1}\right)=C_{k}$ that

$Y_{1}^{2}(\psi, 0)=\frac{1}{I}$

We substitute $Y_{1}$ for $Y$ in Eq. (24), differentiate the obtained equation with respect to $\psi$, take $\psi=\psi_{1}$, and use $\lambda_{1}\left(\psi_{1}\right)=\omega^{2}$ and $V_{\mathrm{A}}\left(\psi_{1}\right)=C_{k}$. As a result we obtain

$\frac{\partial^{3} Y_{1}}{\partial z^{2} \partial \psi}+\frac{\omega^{2}}{C_{k}^{2}} \frac{\partial Y_{1}}{\partial \psi}=-\frac{1}{C_{k}^{2}} \frac{\partial V_{\mathrm{A}}^{2}}{\partial \psi} \frac{\partial^{2} Y_{1}}{\partial z^{2}}-\frac{\mathrm{d} \lambda_{1}}{\mathrm{~d} \psi} \frac{Y_{1}}{C_{k}^{2}}$,

where $\psi=\psi_{1}$. Using Eq. (75) yields

$\left.\frac{\partial V_{\mathrm{A}}^{2}}{\partial \psi}\right|_{\psi=\psi_{1}}=\frac{2 C_{k}^{2}\left(\rho_{\mathrm{i}}-\rho_{\mathrm{e}}\right)}{l B R^{2}\left(\rho_{\mathrm{i}}+\rho_{\mathrm{e}}\right)}$. 
Using this result and Eqs. (24) and (76) we obtain

$$
\frac{\partial^{3} Y_{1}}{\partial z^{2} \partial \psi}+\frac{\omega^{2}}{C_{k}^{2}} \frac{\partial Y_{1}}{\partial \psi}=\left(\frac{2 \omega^{2}\left(\rho_{\mathrm{i}}-\rho_{\mathrm{e}}\right)}{l B R^{2}\left(\rho_{\mathrm{i}}+\rho_{\mathrm{e}}\right)}-\frac{\partial \lambda_{1}}{\partial \psi}\right) \frac{X Y_{1}\left(\psi_{1}, 0\right)}{C_{k}^{2}} .
$$

Multiplying Eq. (80) by $X$, integrating the obtained equation with respect to $z$, and using Eqs. (16), (33) and (50) yields

$$
\Delta=-\left.\frac{\partial \lambda_{1}}{\partial \psi}\right|_{\psi=\psi_{1}}=-\frac{2 \omega^{2} J}{l B R^{2} I}, \quad J=\int_{-L / 2}^{L / 2} \frac{\left(\rho_{\mathrm{i}}-\rho_{\mathrm{e}}\right) X^{2}}{\left(\rho_{\mathrm{i}}+\rho_{\mathrm{e}}\right) C_{k}^{2}} \mathrm{~d} z .
$$

Then using Eqs. (75)-(77) and (81), and the relation $\rho\left(\psi_{1}\right)=$ $\frac{1}{2}\left(\rho_{\mathrm{i}}+\rho_{\mathrm{e}}\right)$ we obtain from Eq. (45)

$\Gamma=\frac{\pi}{4} l \omega^{2}|J|$.

Now, we introduce the dimensionless variables

$Z=\frac{2 z}{L}, \quad \tau=\frac{t}{t_{\text {cool }}}, \quad \varpi=\frac{\omega L}{C_{f}}, \quad \kappa=\frac{L}{\pi H_{0}}, \quad \Lambda=\frac{R}{R_{f}}$,

where the kink speed at the footpoints is defined by

$C_{f}^{2}=\frac{2 \zeta B^{2}(0)}{\mu_{0} \rho_{f}(\zeta+1)}$.

We solve a linear problem. Hence we can fix $A$ at the initial moment arbitrary. If we take $A(0)=1$, then $A(t)$ is the ratio of the current oscillation amplitude and its value at the initial time. Now, we substitute $X$ for $S_{0}$ in Eq. (16). Then, using Eqs. (68), (71)-(74), and the relation $B R^{2}=$ const we obtain

$$
\frac{\partial^{2} X}{\partial Z^{2}}+\frac{\varpi^{2} \Lambda^{4} X}{4(\zeta+1)}\left[\zeta \exp \left(-\kappa \mathrm{e}^{\tau} \cos \frac{\pi Z}{2}\right)+\exp \left(-\kappa \cos \frac{\pi Z}{2}\right)\right]=0
$$

Since the loop is symmetric with respect to the apex point, and we consider the fundamental mode, we can solve Eq. (85) using the boundary conditions

$$
\frac{\partial X}{\partial Z}=0 \quad \text { at } Z=0, \quad X=0 \quad \text { at } Z=1 .
$$

The function $X(Z)$ takes maximum at $Z=0$, so we can reduce the condition $\max _{z} X(z)=1$ to $X(0)=1$. This boundary value problem determines $\varpi$ and $X(Z)$. We recall that $\varpi$ and $X(Z)$ also depend on $\tau$ parametrically.

Using Eqs. (47), (71), (73) and (81)-(83) we obtain

$\frac{\mathrm{d}\left(\varpi \Pi_{+} A^{2}\right)}{\mathrm{d} \tau}=-\alpha \varpi^{2}\left|\Pi_{-}\right| A^{2}$

where

$\Pi_{ \pm}=\int_{0}^{1} X^{2} \Lambda^{4}\left[\zeta \exp \left(-\kappa \mathrm{e}^{\tau} \cos \frac{\pi Z}{2}\right) \pm \exp \left(-\kappa \cos \frac{\pi Z}{2}\right)\right] \mathrm{d} Z$,

$\alpha=\frac{\pi l C_{f} t_{\mathrm{cool}}}{4 L}$

The parameter $\alpha$ determines the relative strength of resonant damping and amplification caused by cooling. We see that the dependence of the oscillation amplitude on time is determined by five dimensionless parameters: $\alpha, \zeta, \kappa, \vartheta$, and $L / L_{c}$.

Numerical solution was obtained using software packages from Wolfram Mathematica 11.3. To obtain the solution to the eigenvalue problem constituted by Eq. (85) with the boundary conditions (86) we used the program NDEigenesystem that calculates the eigenvalue $\varpi(\tau)$ and the eigenfunction $X(\tau, Z)$. Then, we substituted $\varpi(\tau)$ and $X(\tau, Z)$ in Eq. (87) and integrated this equation numerically using the program NIntegrate. By default, this program uses the Global adaptive method that automatically choses a numerical method that minimises the error. As a result, we calculated the dependence of $A$ on $\tau$.

In our calculations we took $\zeta=3$ and $L / L_{c}=6$. The function $A(t)$ is determined numerically for various values of $\alpha, \kappa$ and $\vartheta$. The results of these calculations are presented in Fig. 2 . We see that when there is no resonant absorption $(\alpha=0)$ cooling results in the amplification of oscillations. This result is in a complete agreement with that obtained by Ruderman $(2011 \mathrm{a}, \mathrm{b})$ and Ruderman et al. (2017). As we have already pointed out, our analysis describes competition between the amplification of oscillations due to cooling and damping of oscillations caused by resonance absorption. We see that the magnetic tube expansion enhances the amplification. As a result, when there is no resonant absorption the larger the tube expansion the faster the oscillation amplitude growths. When resonant absorption is present, the larger the tube expansion the slower the decay of the oscillation amplitude is. For particular values of parameters we even can have the oscillation damping in a non-expanding tube, and the oscillation amplification in an expanding tube.

Now, we introduce the critical value of $\alpha$ defined by the condition that $A(1)=A(0)$ for $\alpha=\alpha_{c}$. That means that oscillation amplitude at $t=t_{\text {cool }}$ is equal to its initial amplitude. Similarly, we introduce the critical value of transitional layer thickness, $l_{c}$, given by

$l_{c}=\frac{4 \alpha_{c} L}{\pi C_{f} t_{\mathrm{cool}}}$.

When $l=l_{c}$ the damping due to resonant absorption is balanced by amplification due to cooling.

Figure 3 shows the dependence of $\alpha_{c}$ on $\vartheta$ for various values of $\kappa$. Again, we see that the tube expansion enhances the effect of the amplitude amplification. What is also interesting, the dependence of this effect efficiency on $\kappa$ is not monotonic. The effect efficiency increases when $\kappa$ changes from 0.5 to 1 , and then is getting weaker when $\kappa$ further increases.

The observations of large-amplitude kink oscillations of cooling coronal magnetic loops that do not decay are very scarce. Probably, one of the best documented observations of this kind is that reported by Aschwanden \& Schrijver (2011). Ruderman (2011a) discussed the possibility to explain the undamped nature of this oscillation by the effect of cooling. His conclusion was that cooling can stop damping due to resonant absorption only if the transitional layer is very thin, $l \approx 0.02$. We now discuss how the account of loop expansion can change this estimate.

The initial oscillation period of the oscillations was $395 \mathrm{~s}$. Using the data reported by Aschwanden \& Schrijver (2011) and Ruderman (2011b) obtained that $\kappa \approx 1$ and $t_{\text {cool }} \approx 2050 \mathrm{~s}$. We take the tube expansion $\theta=1.5$ because the effect of expansion is the strongest for this value of $\theta$. The phase speed $C_{k}$ varies along the loop due to the density and cross-section radius variation. The density decrease and cross-section radius increase act in the opposite directions. Hence, we can expect that the variation of $C_{k}$ along the loop is weak and we can take $C_{k} \approx C_{f}$. Then, the oscillation period is approximately equal to $2 L / C_{f}$ and we obtain 

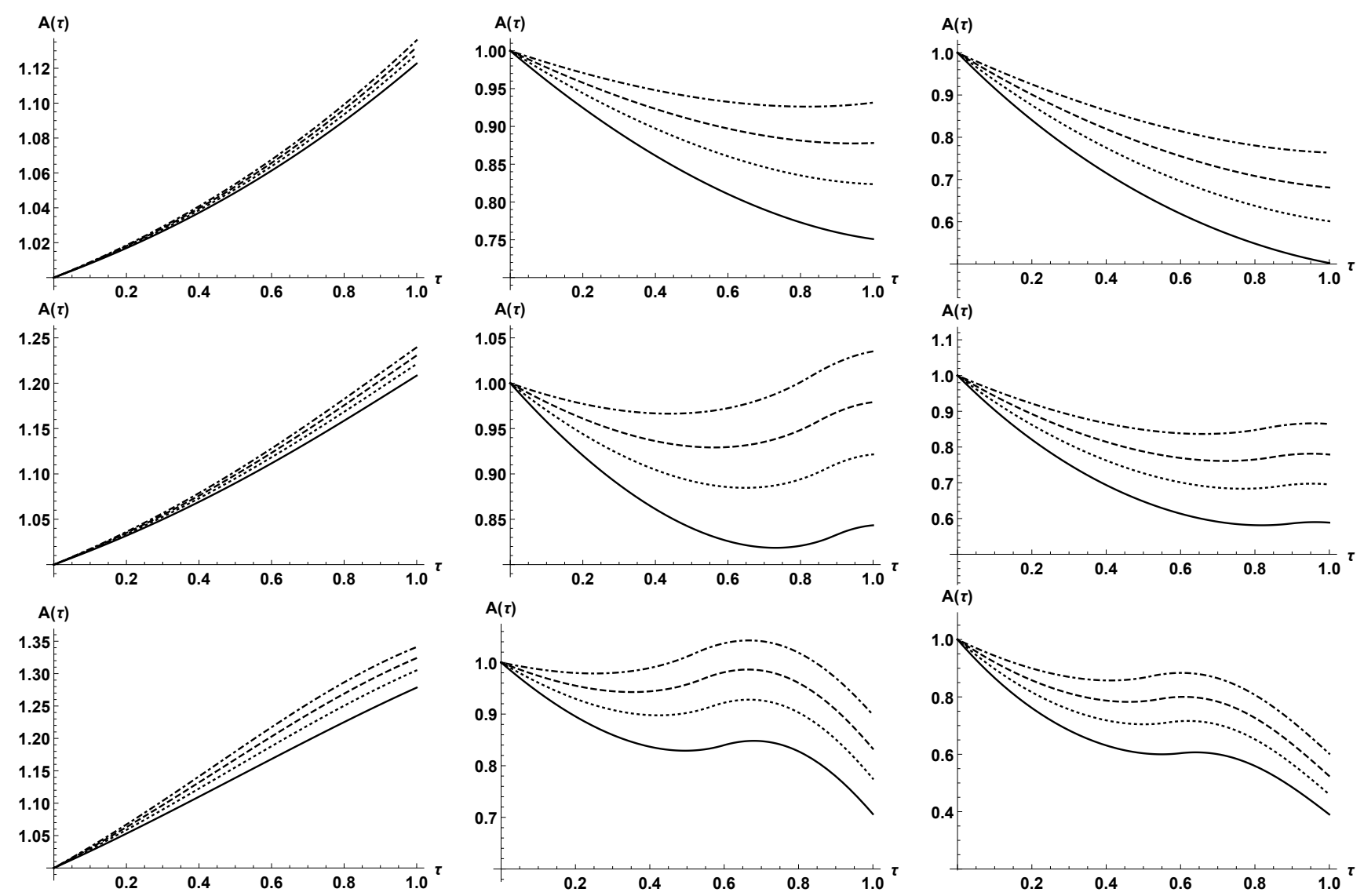

Fig. 2. Dependence of dimensionless amplitude $A$ of the fundamental mode on the dimensionless time $\tau$ for $\zeta=3$ and $L / L_{c}=6$. The upper, middle, and lower panels correspond to $\kappa=0.5,1$, and 2 , respectively. The left, middle, and right panels correspond to $\alpha=0,0.5$, and 1 , respectively. The solid, dotted, dashed, and dashed-dotted lines correspond to $\vartheta=1,1.15,1.3$, and 1.5.

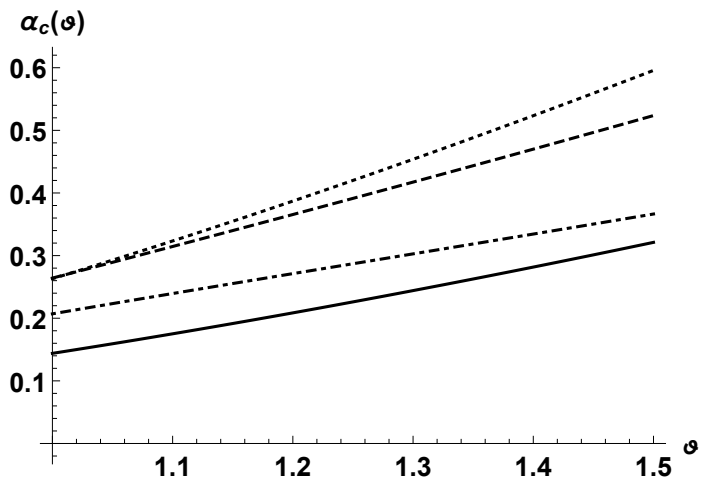

Fig. 3. Dependence of critical value $\alpha_{c}$ on expansion factor $\vartheta$. The solid, dotted, dashed, and dash-dotted lines correspond to $\kappa=0.5,1,1.5$, and 2 , respectively.

$L / C_{f} \approx 198$ s. Using Fig. 3 we obtain that $\alpha_{c} \approx 0.6$. Then it follows from Eq. (90) that $l_{c} \approx 0.074$. Although this is still a very thin transitional layer, it looks more realistic than $l_{c} \approx 0.02$ obtained by Ruderman (2011a).

\section{Summary and conclusions}

In this paper, we studied resonant damping of kink oscillations of cooling coronal magnetic loops. A coronal magnetic loop is modelled by a thin straight magnetic tube with the plasma density and the cross-section radius varying along the tube. The equilibrium plasma density is approximately independent of the radial coordinate inside the core of the tube and outside of the tube. However, it varies in the radial direction from its value in the core region to its value outside of the tube in a thin transitional value. This density variation results in the presence of resonant absorption. We use the system of two equations describing oscillations of non-stationary magnetic tubes in the presence of resonance absorption in the thin tube approximation that were derived by Ruderman et al. (2017). This system contains three dependent variables: the tube displacement $\eta$, and the jumps of the plasma displacement and the magnetic pressure perturbation across the transitional layer.

The system is not closed. To close it, we need to express the jumps of the plasma displacement and the magnetic pressure perturbation in terms of $\eta$. To do this, we applied the thin boundary layer approximation. Shukhobodskiy \& Ruderman (2018) have carried out such a study, in the case of static magnetic tubes, under the assumption that the density in the transitional layer can be factorised and written as a product of two functions, one depending on the coordinate along the tube and the other depending on the magnetic flux function. However, we cannot make this assumption here, when a loop is dynamic, because even if this condition is initially satisfied it will not be valid later because the density is changing with time. Hence, we adapted the derivation given by Shukhobodskiy \& Ruderman (2018). Eventually, we derived an equation describing the evolution of the oscillation amplitude. This equation reduces to the conservation of an adiabatic invariant previously derived by Ruderman et al. (2017) where there is no resonant absorption. 
We studied the amplitude evolution of kink oscillation of a cooling coronal magnetic loop. When doing this, we assumed that the cooling is slow meaning that the cooling time is substantially larger than the oscillation period. We also assumed that cooling only occurs in the magnetic tube, while the temperature of the outside plasma does not change. The cooling causes the amplification of loop oscillation, while resonant absorption causes its decay. Hence, the governing equation for the oscillation amplitude describes the competition between the amplification and damping. This equation was solved numerically. Our main aim was to study the effect of the loop expansion on the amplitude evolution of transversal oscillations. We found that the loop expansion acts in favour of oscillation amplification. When there is no damping due to resonant absorption the larger the loop expansion the faster the oscillation amplitude growths. If resonant absorption is present then the loop expansion either reduces the damping rate, or even can turn it into the amplification of oscillation.

We also considered the possibility of existence of oscillations that are not amplified and also do not decay. We define such oscillations as those with the amplitude at the cooling time $t_{\text {cool }}$ equal to its initial value. In this case, there is the balance between the amplification due to cooling and decay due to resonant absorption. Again, we found that the loop expansion acts in favour of amplification. As a result, the larger the loop expansion the stronger resonant absorption can be counterbalanced by cooling with the same cooling rate.

Acknowledgements. M.S.R. and R.E. acknowledge the support from the Science and Technology Facilities Council (STFC, grant nr ST/M000826/1) grant.

\section{References}

Abedini, A. 2018, Sol. Phys., 293, 22

Al-Ghafri, K. S., Ruderman, M. S., Williamson, A., \& Erdélyi, R. 2014, ApJ, 786,36
Aschwanden, M. J., Fletcher, L., Schrijver, C. J., \& Alexander, D. 1999, ApJ, 520,880

Aschwanden, M. J., \& Schrijver, C. J. 2011, ApJ, 736, 102

Aschwanden, M. J., \& Terradas, J. 2008, ApJ, 686, L127

Bender, C. M., \& Orszag, S. A. 1978, 1978, Advanced Mathematical Methods for Scientists and Engineers (New York: McGraw-Hill)

Coddington, E. A., \& Levinson, N. 1955, Theory of Ordinary Differential Equations (New York: McGraw-Hill)

Duckenfield, T., Anfinogentov, S. A., Pascoe, D. J., \& Nakariakov, V. M. 2018, ApJ, 854, L5

Dymova, M. V., \& Ruderman, M. S. 2005, Sol. Phys., 229, 79

Dymova, M. V., \& Ruderman, M. S. 2006, A\&A, 457, 1059

Edwin, P. M., \& Roberts, B. 1983, Sol. Phys., 88, 179

Erdélyi, R., \& Taroyan, Y. 2008, A\&A, 489, L49

Goossens, M., Andries, J., \& Aschwanden, M. J. 2002, A\&A, 394, L39

Goossens, M., Erdélyi, R., \& Ruderman, M. S. 2011, Space Sci. Rev., 158, 289

Goossens, M., Hollweg, J. V., \& Sakurai, T. 1992, Sol. Phys., 138, 233

Hollweg, J. V., \& Yang, G. 1988, Phys. Rep., 93, 5423

Klimchuk, J. 2000, A., 193, 53

Morton, R. J., \& Erdélyi, R. 2009, ApJ, 707, 750

Morton, R. J., \& Erdélyi, R. 2010, A\&A, 519, A43

Naimark, M. A. 1967, Linear Differential Equations, Part I (New York: Frederic Ungar Publ. Co.)

Nakariakov, V. M., Ofman, L., Deluca, E. E., Roberts, B., \& Davila, J. M. 1999, Science, 285,862

Ruderman, M. S. 2011a, A\&A, 534, A78

Ruderman, M. S. 2011b, Sol. Phys., 271, 41

Ruderman, M. S., \& Erdélyi, R. 2009, Space Sci. Rev., 149, 199

Ruderman, M. S., \& Roberts, B. 2002, ApJ, 577, 475

Ruderman, M. S., Shukhobodsky, A. A., \& Erdélyi, R. 2017, A\&A, 602, A50

Ruderman, M. S., Verth, G., \& Erdélyi, R. 2008, ApJ, 686, 694

Ryutov, D. D., \& Ryutova, M. P. 1976, Sov. Phys. - JETP, 43, 491

Shukhobodskiy, A. A., \& Ruderman, M. S. 2018, A\&A, 615, A156

Su, W., Guo, Y., Erdélyi, R., et al. 2018, Nat. Sci. Rep., 8, 4471

Terradas, S., Oliver, R., \& Ballester, J. L. 2006, ApJ, 650, L91

Tsuneta, S., Ichimoto, K., Katsukawa, Y., et al. 2008, ApJ, 688, 1374

Van Doorsselaere, T., Andries, J., Poedts, S., \& Goossens, M. 2004a, ApJ, 606, 1223

Van Doorsselaere, T., Debosscher, A., Andries, J., \& Poedts, S. 2004b, A\&A, 424, 1065

Verth, G., \& Erdélyi, R. 2008, A\&A, 486, 1015

Watko, J. A., \& Klimchuk, J. A. 2000, Sol. Phys., 193, 77 